Supporting information

\title{
Ultrafast Self-Assembly of Bottlebrush Statistical Copolymers: Well Ordered Nanostructures from One-Pot Polymerizations
}

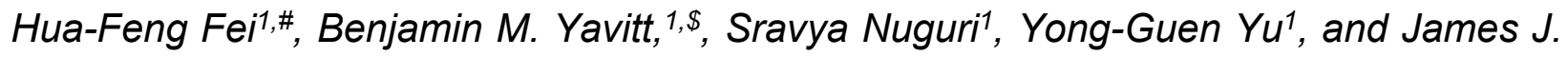
Watkins $^{1 *}$

${ }^{1}$ Department of Polymer Science and Engineering, University of Massachusetts Amherst, 120 Governors Drive, Amherst, MA, 01003 United States

Corresponding author:

(J.J.W.) *E-mail: watkins@polysci.umass.edu

\section{Experimental}

Materials. Exo-5-norbornenecarboxylic acid (>97\%), 2-Hydroxyethyl 2-Bromoisobutyrate (>99.0), $\quad \mathrm{N}, \mathrm{N}, \mathrm{N}$ ',N"',N"-Pentamethyldiethylenetriamine $\quad$ (PMDETA, 99\%), N,N'dicyclohexyl-carbodiimide $\quad(\mathrm{DCC}, \quad>99.0 \%), \quad$ Triethylamine $\quad$ (TEA, 99.5\%), 4 dimethylaminopyridine (DMAP, $>99 \%$ ), Copper bromide and Grubbs $3^{\text {rd }}$ generation catalyst were purchased from Sigma-Aldrich, and used without any further purification. Anhydrous toluene (99.8\%), anhydrous dichloromethane (DCM), and anhydrous tetrahydrofuran (THF, 99.9\%) were provided from Acros Organics. Styrene was supplied from Sigma-Aldrich and passed through basic aluminum oxide before polymerization. Hydroxy terminated polydimethylsiloxane (PDMS-OH, $M_{n}=4670 \mathrm{~g} / \mathrm{mol}$ ) was purchased from Sigma-Aldrich and heated at $T=120^{\circ} \mathrm{C}$ for about $4 \mathrm{~h}$ under nitrogen flow to remove 
moisture before used. The hydroxy terminated $\mathrm{PS}_{0.9 \mathrm{k}}, \mathrm{PS}_{2 \mathrm{k}}, \mathrm{PDMS}_{2 \mathrm{k}}$ and $\mathrm{PDMS}_{0.8 \mathrm{k}}$ were purchased from Polymer Source Inc.

Measurements. Proton nuclear magnetic resonance $\left({ }^{1} \mathrm{H}\right.$ NMR) spectroscopy was conducted in deuterated chloroform using a Bruker 500 NMR spectrometer. Gel permeation chromatography (GPC) of the bottlebrushes was carried out in THF +1 vol. \% triethylamine (TEA) using two PLgel $10 \mu \mathrm{m}$ mixed-B LS columns (Polymer Laboratories) connected in series with a Wyatt Technologies DAWN EOS multi-angle laser light scattering (MALLS) detector and RI detector at a flow rate of $1.0 \mathrm{ml} / \mathrm{min}$. No calibration standards were used as direct $\mathrm{dn} / \mathrm{dc}$ values were obtained for each injection by assuming $100 \%$ mass elution from the columns. GPC of macromonomers and polymerization kinetic research was carried out using a Polymer Laboratories PL-GPC50 instrument with two $5 \mu \mathrm{m}$ mixed-D columns, a $5 \mu \mathrm{m}$ guard column, and a RI detector (HP1047A). THF was used as the eluent at a flow rate of $1.0 \mathrm{~mL} / \mathrm{min}$. Polystyrene standards were used for the calibration. Small-angle X-ray scattering (SAXS) measurements were conducted at the Complex Material Scattering (CMS) beamline (11-BM, NSLS II, Brookhaven National Lab). Scattering patterns were collected on a Dectrix $2 \mathrm{M}$ detector (pixel size $=75 \mu \mathrm{m} \times$ $75 \mu \mathrm{m}$ ) at a sample to detector distance of $2 \mathrm{~m}$ using an X-ray beam with the energy of $13.5 \mathrm{keV}(\lambda=0.092 \mathrm{~nm})$. Bulk samples were placed into a circular mold and sandwiched on each side with Kapton tape. The measurement exposure time was set to 15 seconds. Additional SAXS experiments were performed on a Ganesha SAXS-LAB using $0.154 \mathrm{~nm}$ (Cu- $\mathrm{K}_{\alpha}$ radiation), and X-ray beam area of approximately $0.04 \mathrm{~mm}^{2}$. Transmission electron microscopy (TEM) measurements were conducted with a JEOL 2000FX TEM 
operated at an accelerating voltage of $200 \mathrm{kV}$. Thin sections of approximately $40 \mathrm{~nm}$ in thickness were prepared using a Leica Ultracut UCT microtome equipped with a Leica EM FCS cryogenic sample S2 chamber operated at $\mathrm{T}=-160^{\circ} \mathrm{C}$.

Synthesis of norbornene-terminated polystyrene macromonomer (PS-NB MM) (Scheme S1) ${ }^{1}$. A Schlenk tube was charged with $\mathrm{CuBr}(0.310 \mathrm{~g}, 2.16 \mathrm{mmol})$, PMDETA (0.375 g, $2.16 \mathrm{mmol})$, styrene $(22.55 \mathrm{~g}, 216.83 \mathrm{mmol})$ and 2-Hydroxyethyl 2Bromoisobutyrate $(0.456 \mathrm{~g}, 2.16 \mathrm{mmol})$ was used as the initiator. Three freeze-pumpthaw cycles were performed, and the tube was stirred in an oil bath at $\mathrm{T}=110{ }^{\circ} \mathrm{C}$ for 30 min. After the allotted time, the mixture was diluted with tetrahydrofuran (THF). The copper complex was removed by passing through a neutral alumina column, and THF was removed by rotary evaporation. The mixture was precipitated in methanol ( 3 times) and the solid was collected after filtration and dried at room temperature in a vacuum overnight. Hydroxyl-terminated polystyrene (PS-OH) with molecular weight $M_{\mathrm{n}}=2.8$ $\mathrm{kg} / \mathrm{mol}$ (measured by GPC) was obtained.

PS-OH (5.6 g, $2.0 \mathrm{mmol}), \mathrm{N}, \mathrm{N}^{\prime}$-dicyclohexyl-carbodiimide (DCC) $(3.1 \mathrm{~g}, 15 \mathrm{mmol})$, and exo-5-norbornenecarboxylic acid $(0.55 \mathrm{~g}, 4.0 \mathrm{mmol})$ were added into a dry flask followed by $30 \mathrm{~mL}$ of anhydrous DCM. 4-dimethylaminopyridine (DMAP) $(0.10 \mathrm{~g}, 0.82 \mathrm{mmol})$ was used as catalyst. The reaction was carried out at room temperature and stirred under nitrogen overnight. The solution was filtered; the filtrate was evaporated and then precipitated in methanol (3 times). The white solid was collected after filtration and dried at room temperature in a vacuum overnight. Norbornene-terminated polystyrene (PS-NB MM) $(4.7 \mathrm{~g})$ with molecular weight $M \mathrm{n}=2.9 \mathrm{~kg} / \mathrm{mol}$ (measured by $\mathrm{GPC}$ and ${ }^{1} \mathrm{H} \mathrm{NMR}$ ) was obtained. The other molecular weight polystyrene are synthesized in similar method. 
Synthesis of norbornene-terminated PDMS macromonomer (PDMS-NB MM) (Scheme S2)2. PDMS-OH (9.34 g, 2.0 mmol), N,N'-dicyclohexyl-carbodiimide (DCC) (3.1

$\mathrm{g}, 15 \mathrm{mmol})$, and exo-5-norbornenecarboxylic acid $(0.55 \mathrm{~g}, 4.0 \mathrm{mmol})$ were added into a dry flask followed by $30 \mathrm{~mL}$ of anhydrous DCM. 4-dimethylaminopyridine (DMAP) (0.10 $\mathrm{g}, 0.82 \mathrm{mmol}$ ) was used as catalyst. The reaction was carried out at room temperature and stirred under nitrogen overnight. After the reaction mixture was filtered, the solvent of product (PDMS-NB MM) was evaporated and washed with methanol at least 5 times until all the impurities were removed completely, as confirmed by ${ }^{1} \mathrm{H}$ NMR. Excess solvent was evaporated under nitrogen flow, finally a transparent viscous liquid (PDMS-NB MM, $8.5 \mathrm{~g}$ ) was obtained. The NB-PS ${ }_{0.9 \mathrm{k}}$, NB-PS ${ }_{2 \mathrm{k}}, \mathrm{NB}-\mathrm{PDMS}_{2 \mathrm{k}}$ and NB-PDMS $\mathrm{S}_{0.8 \mathrm{k}}$ were synthesized in analogous steps.

\section{Bottlebrush statistical copolymer synthesis through one shot ROMP.}

In a typical experiment, $50 \mathrm{mg}$ of each macromonomer was added to the same vial, followed by the $1.5 \mathrm{~mL}$ of anhydrous DCM. The reaction was carried out in the glovebox. At room temperature, the copolymerization was initiated by adding desired amount of 3rd generation Grubbs catalyst solution in DCM. This solution was stirred for an additional 2-3 hours. The reaction was quenched with ethyl vinyl ether. The complete conversion of both PS-NB and PDMS-NB MM was confirmed quantitatively from ${ }^{1} \mathrm{H}$ NMR spectra, the signal from protons on the terminal bonds of NB end groups were absent for all the bottlebrushes $(\delta=6.15-6.28 \mathrm{ppm})$. For the polymerization kinetic research, when the macromonomers mixed in equals amounts, both macromonomers smoothly copolymerized to completion in 20 min (Figure S1 b). GPC analysis of aliquots taken at different reaction times allowed us to monitor the consumption of each macromonomer 
independently by watching the disappearance of the two monomers. The two monomers almost have the same consumption rates and after copolymerization of $20 \mathrm{~min}$, those two monomers were eventually totally consumed at the same time. Thus, the produced random bottlebrush copolymers had a PS side chain and a PDMS side chain statistical distribution on the backbone as illustrated in the cartoon in Figure 1. The GPC analysis of the final copolymers evidenced almost complete disappearance of the starting macromonomers, and the formation of the brush block copolymers with low dispersity (PDI < 1.3, Table 1), consistent with the living nature of ROMP.

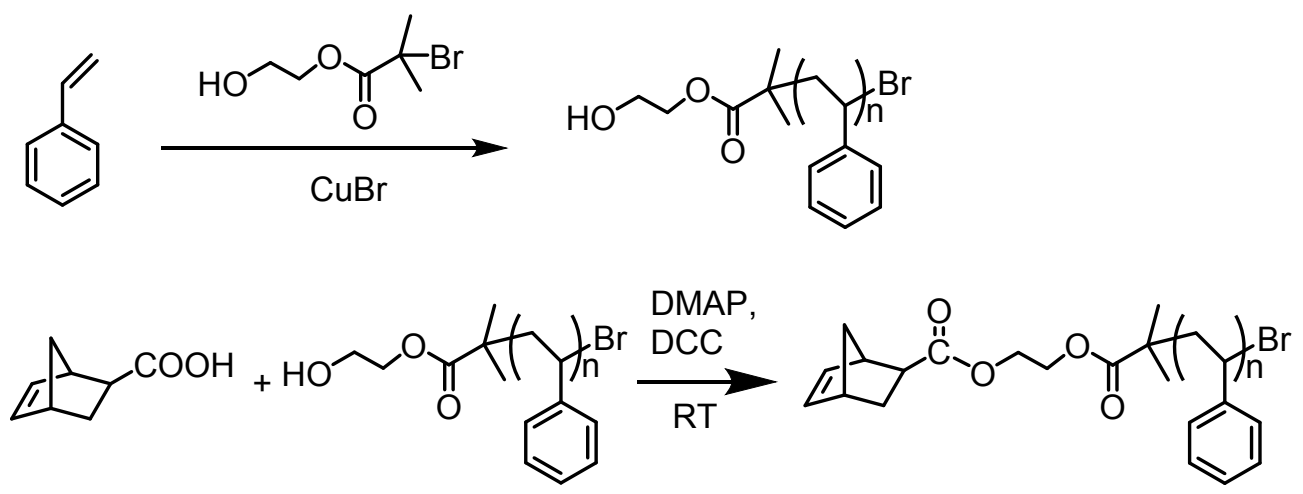

Scheme S1. Synthesis of PS-NB Macromonomer via ATRP polymerization and norbornene functionalization.

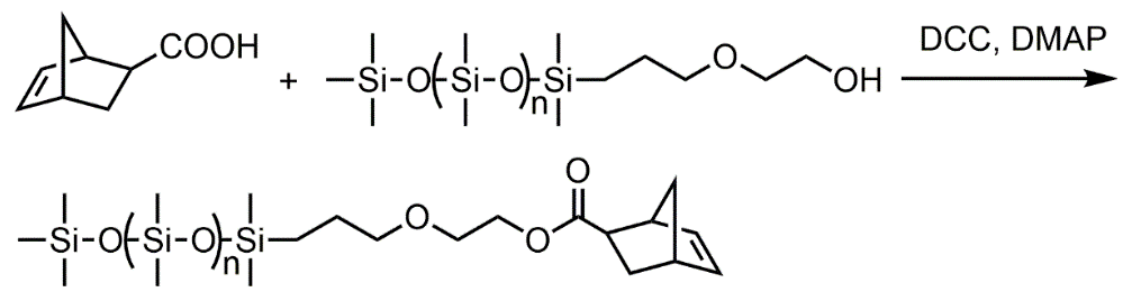

Scheme S2. Synthesis of PDMS-NB macromonomer by norbornene functionalization of hydroxy terminated PDMS. 
a<smiles>CC(C)(C(=O)OCCOCC[Si](C)(C)O[Si](C)(C)O[Si](C)(C)C)C(=O)OCCOC(=O)C1CC2C=CC1C2</smiles>

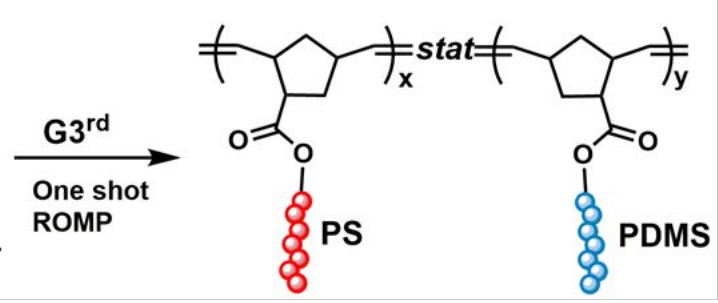

b

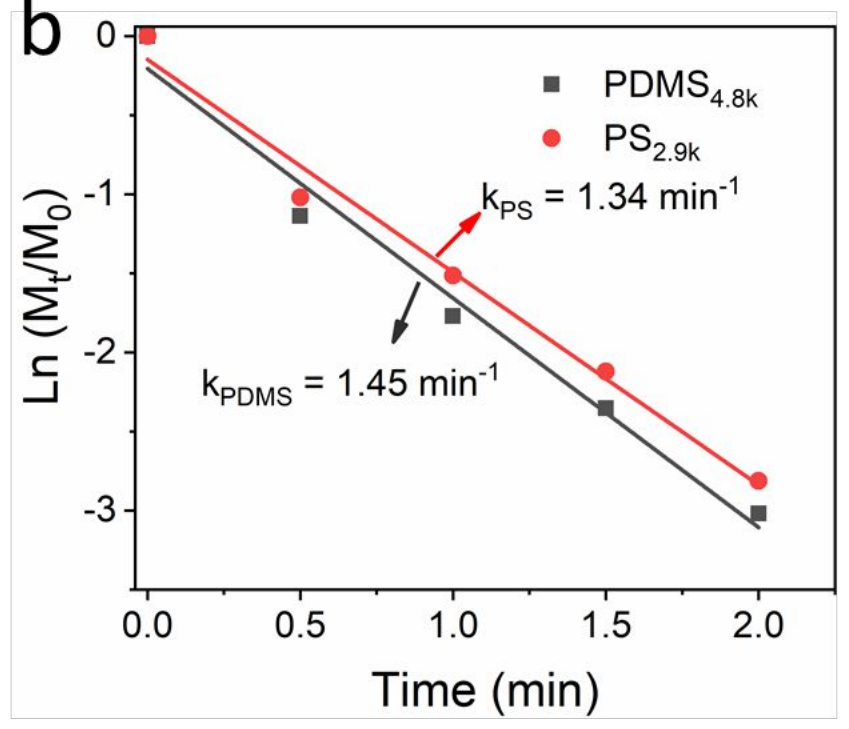

C

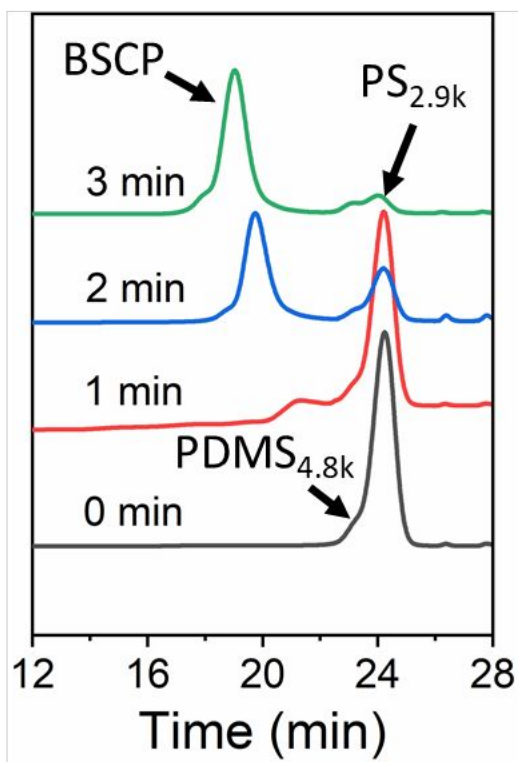

Figure S1. A) Reaction scheme for one-shot ROMP copolymerization of PS-MM and PDMS-MM to form PS-stat-PDMS bottlebrush statistical copolymers. B) Kinetic studies of homopolymerization of $\mathrm{PS}_{2.9 \mathrm{k}}$ and $\mathrm{PDMS}_{4.8 \mathrm{k}}$. Reactivity constants were measured at a monomer concentration of $\sim 50 \mathrm{mg} / \mathrm{mL}$, i.e. $100 \mathrm{mg}$ macromonomer was dissolved into 2 $\mathrm{mL}$ DCM, and catalyst concentration of $\sim 0.15 \mathrm{mg} / \mathrm{mL}$. C) Time resolved gel permeation chromatography (GPC) showing conversion of MMs into BSCPs. 


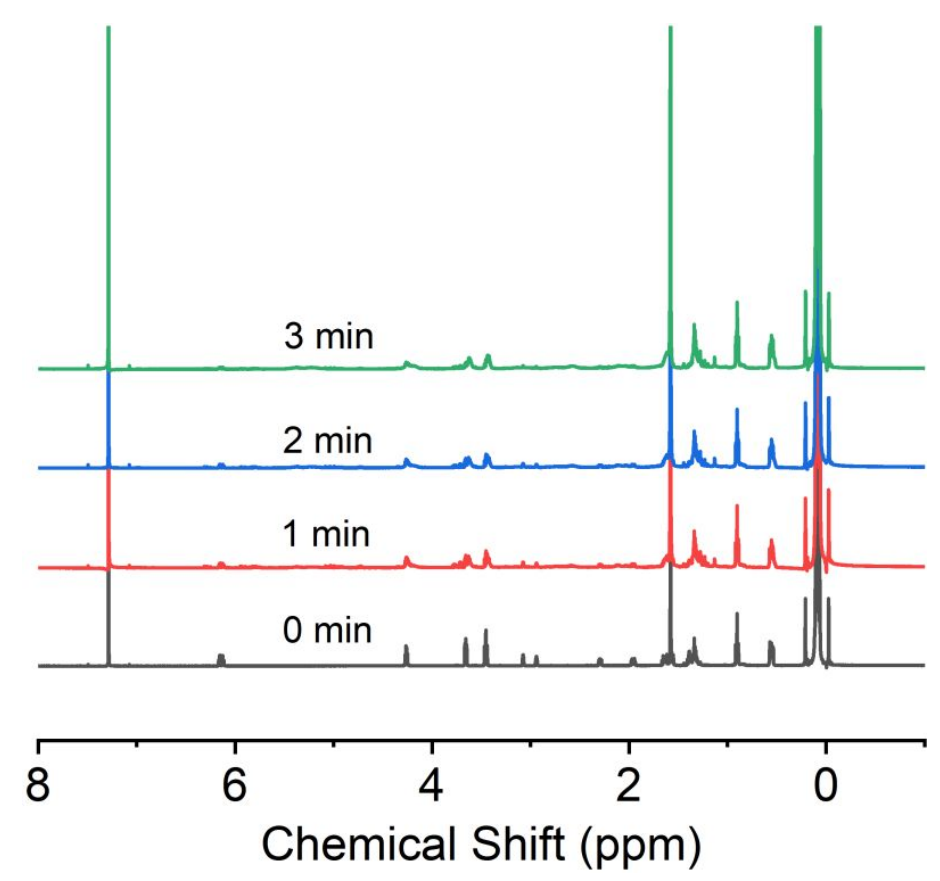

Figure S2. ${ }^{1} \mathrm{H}$ NMR polymerization kinetics monitoring for PDMS-NB.

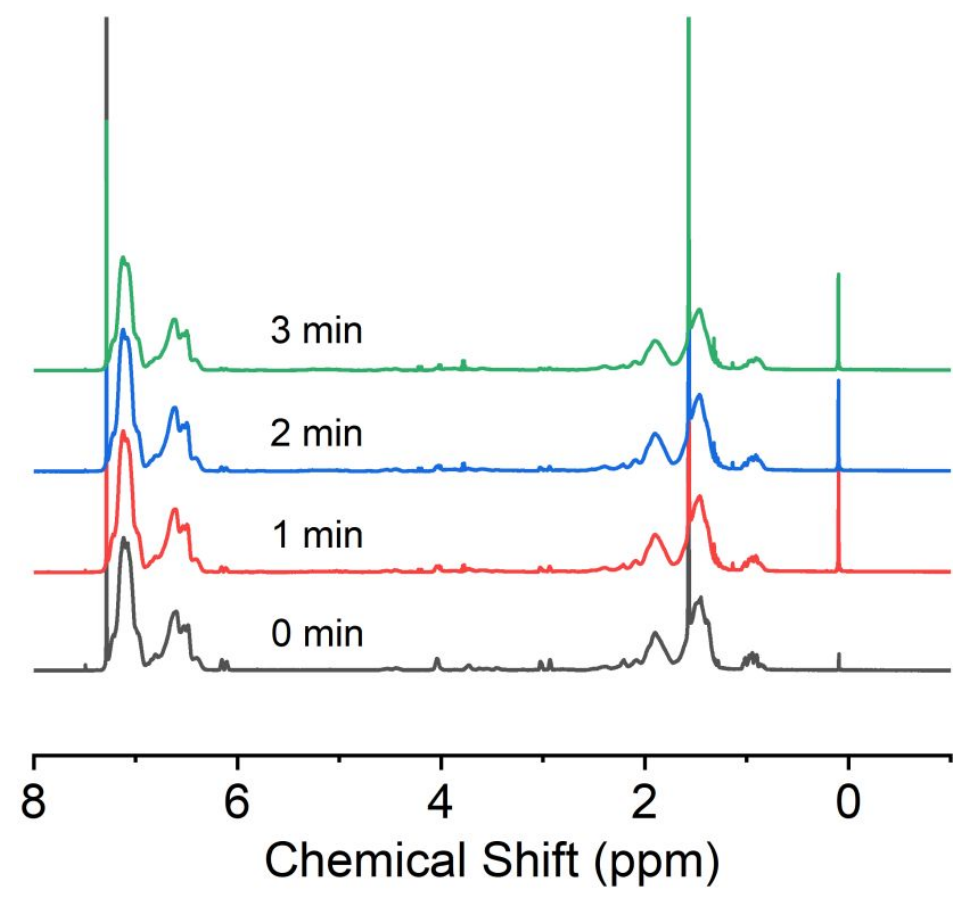

Figure S3. ${ }^{1} \mathrm{H}$ NMR polymerization kinetics monitoring for PS-NB. 


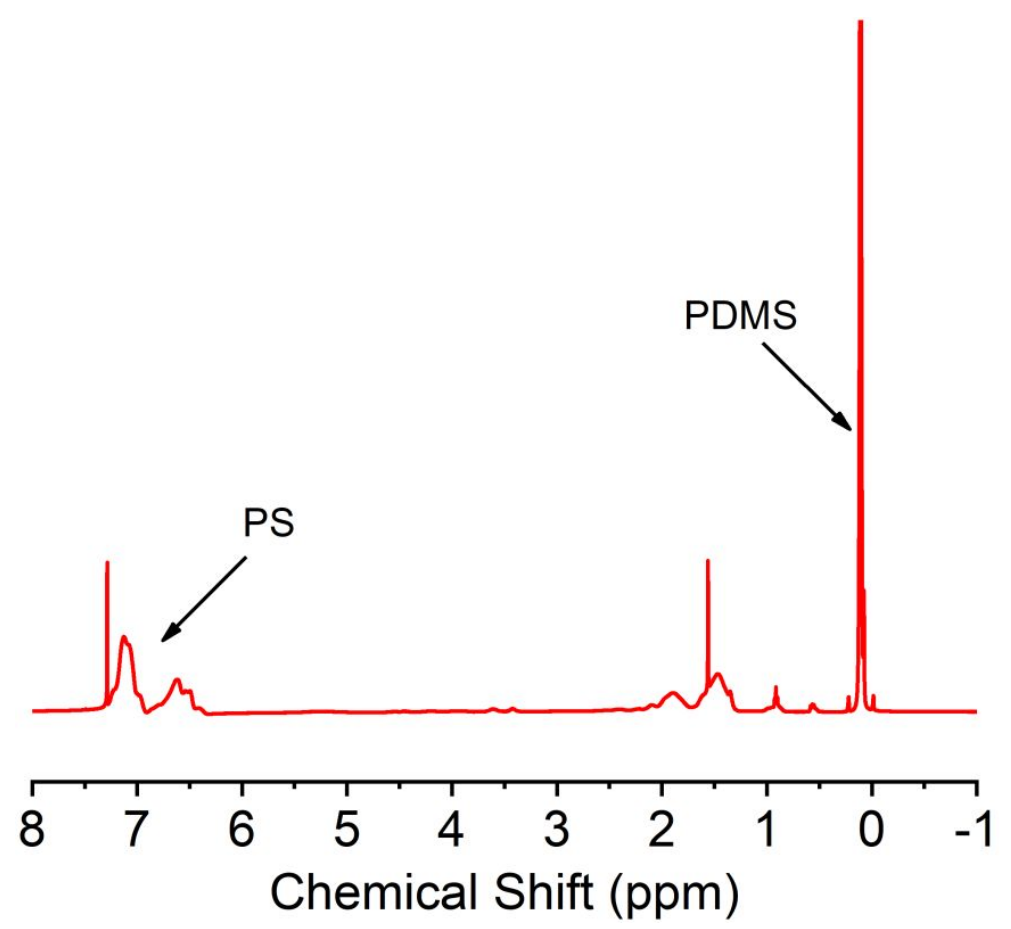

Figure S4. ${ }^{1} \mathrm{H}$ NMR for typical BSCP. 6.30 to $7.25 \mathrm{ppm}$ are assigned to proton of benzene on the polystyrene, 0 to 0.3 ppm are assigned to methyl proton on PDMS.

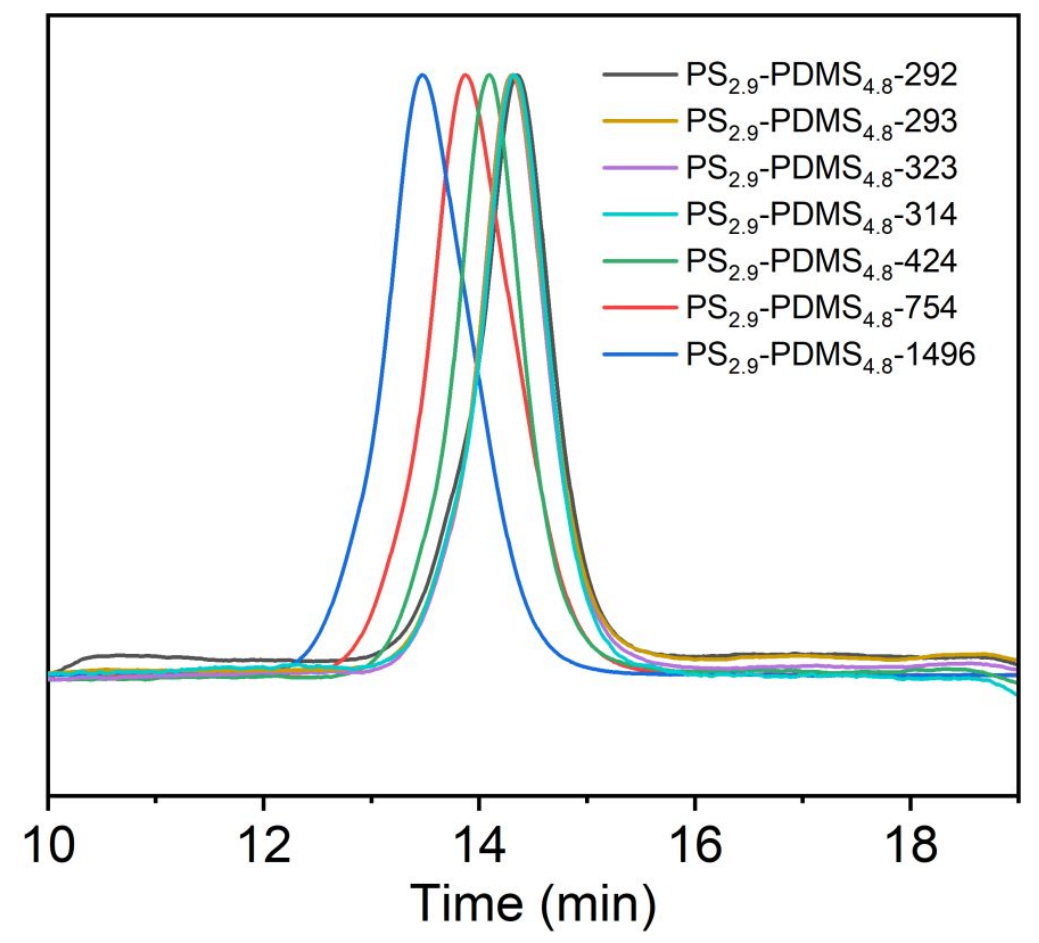

Figure S5. GPC MALLS traces of the BSCP. 


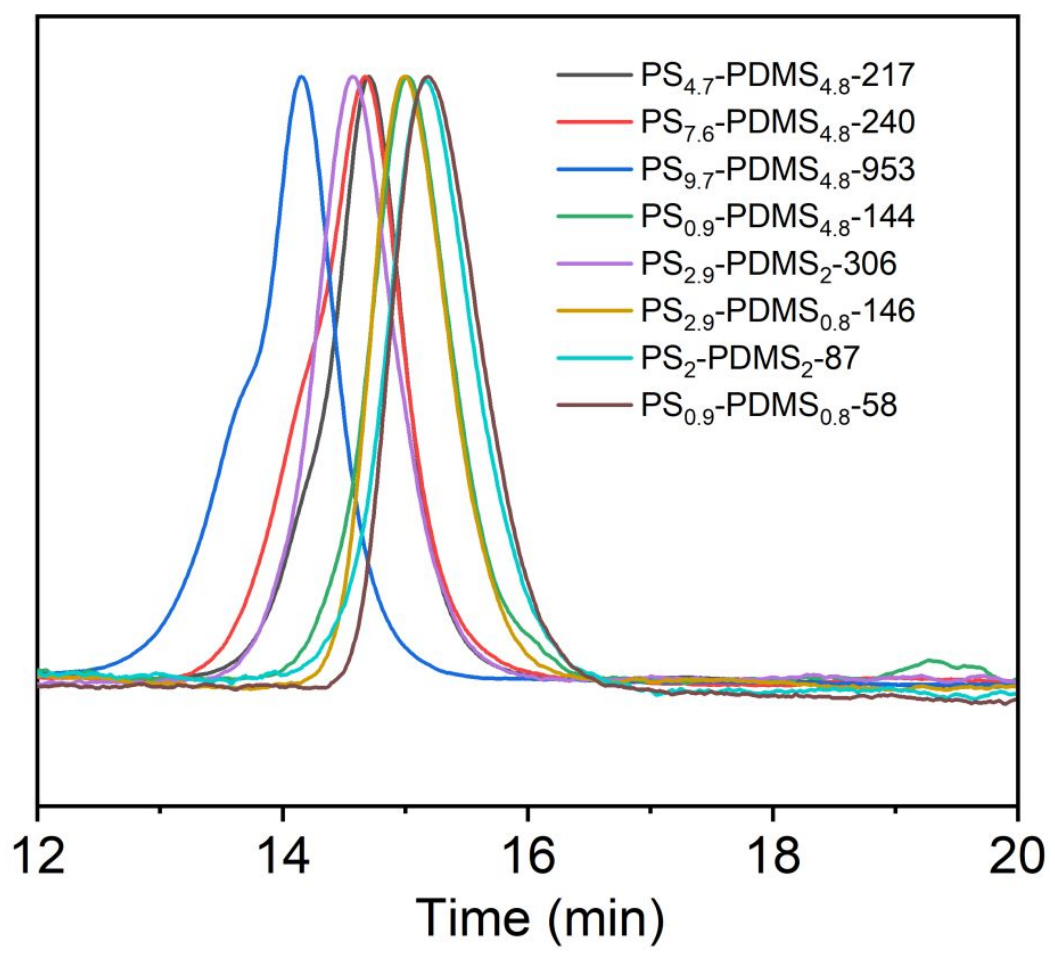

Figure S6. GPC MALLS traces of the BSCP.

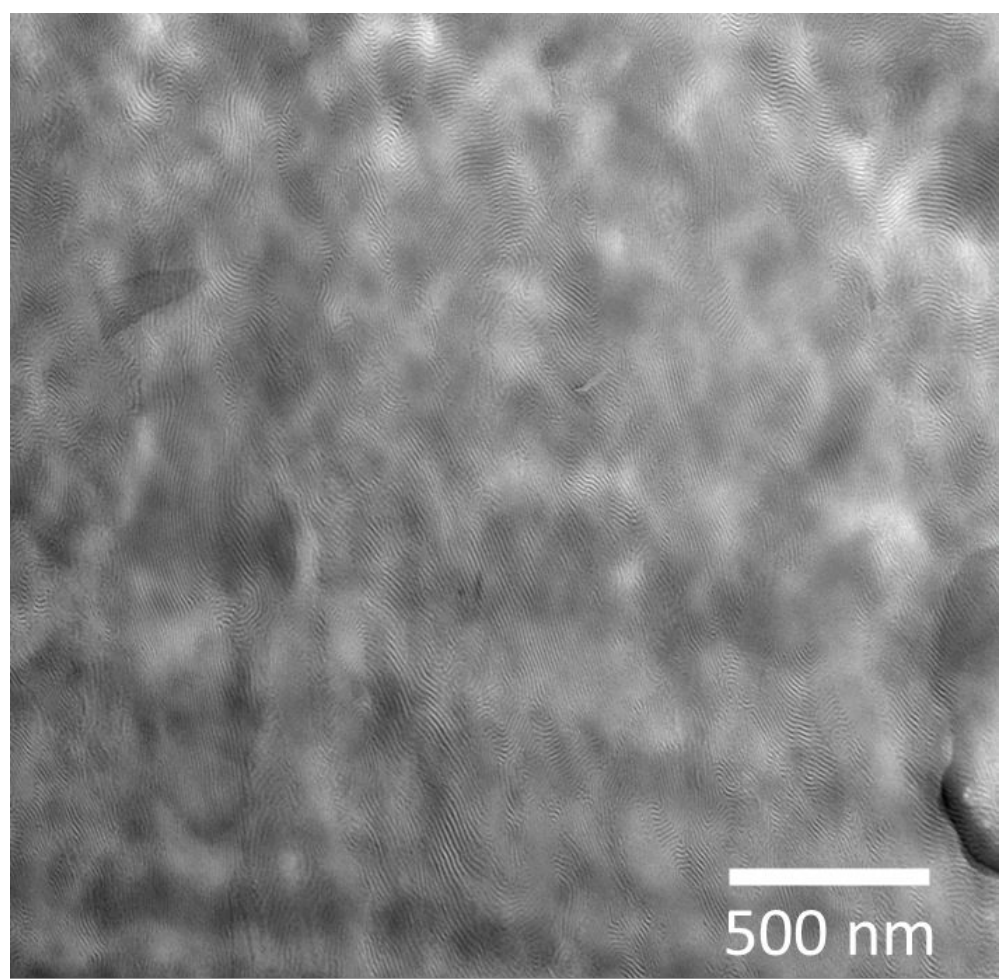

Figure S7. Large area TEM image of $\mathrm{PS}_{2.9} \mathrm{PDMS}_{4.8}-293$. 


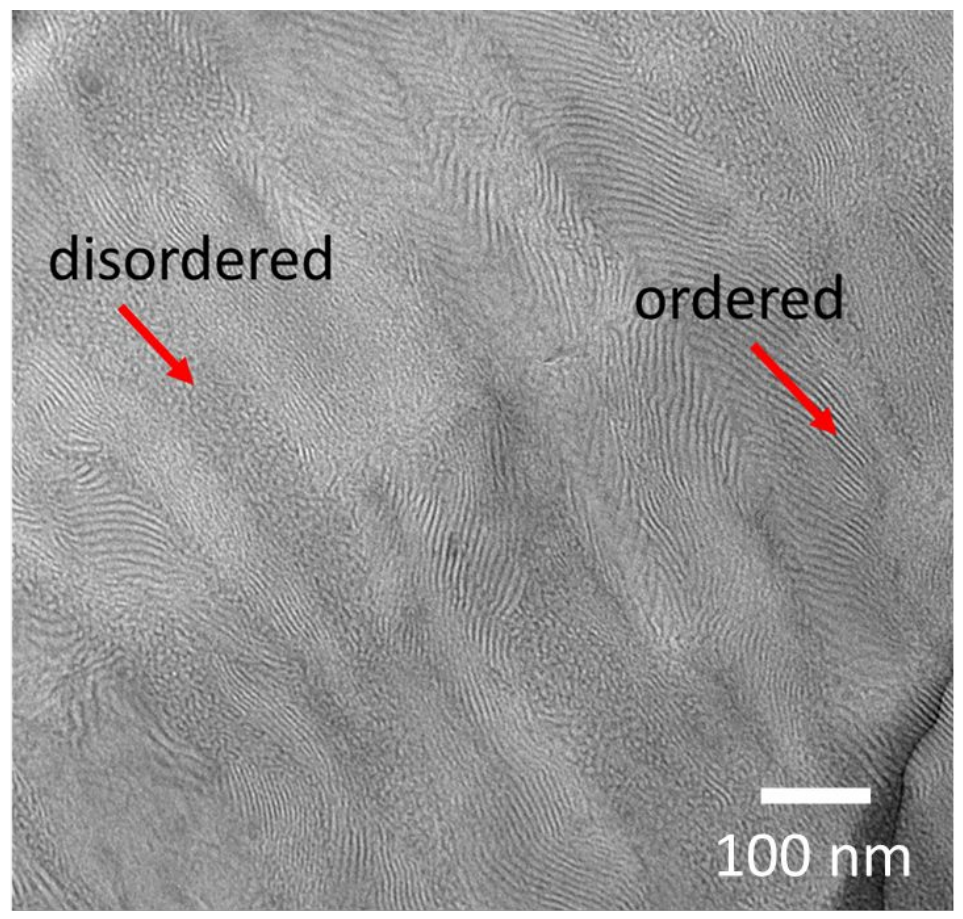

Figure S8. Large area TEM image of $\mathrm{PS}_{2.9}-\mathrm{PDMS}_{4.8}-323$. Regions of well-ordered lamellae and disordered wormlike morphologies are observed.

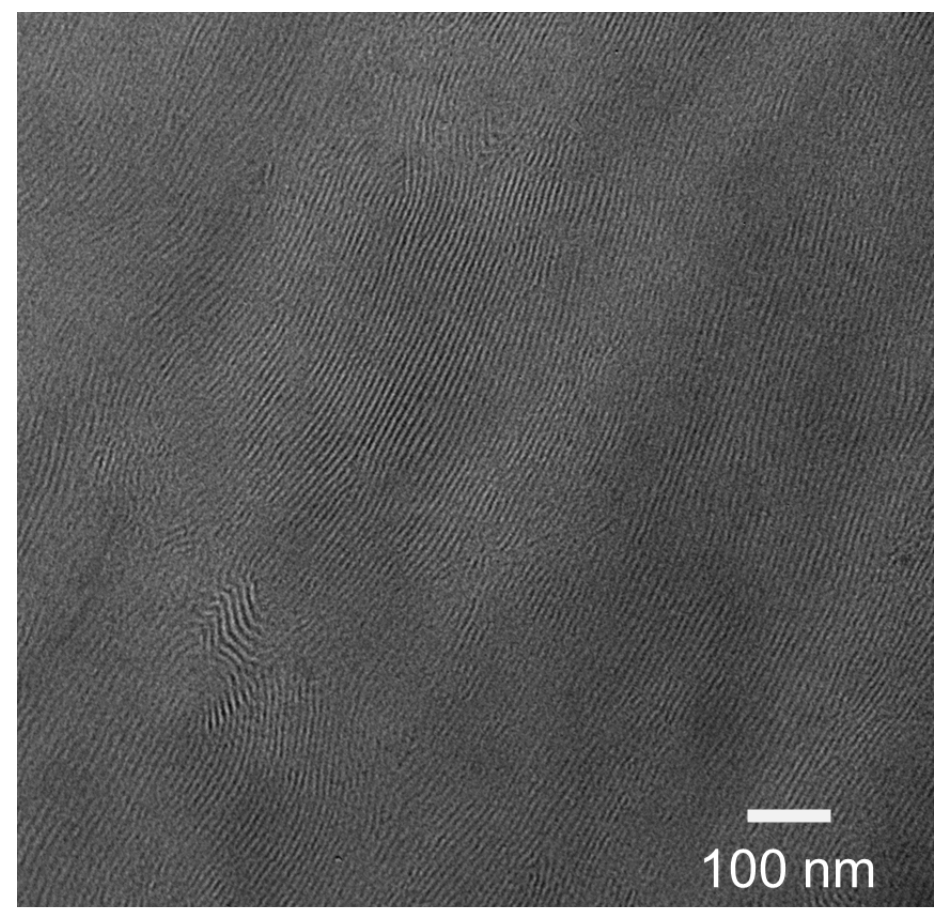

Figure S9. Large area TEM image of $\mathrm{PS}_{0.9}-\mathrm{PDMS}_{4.8}-144$ 


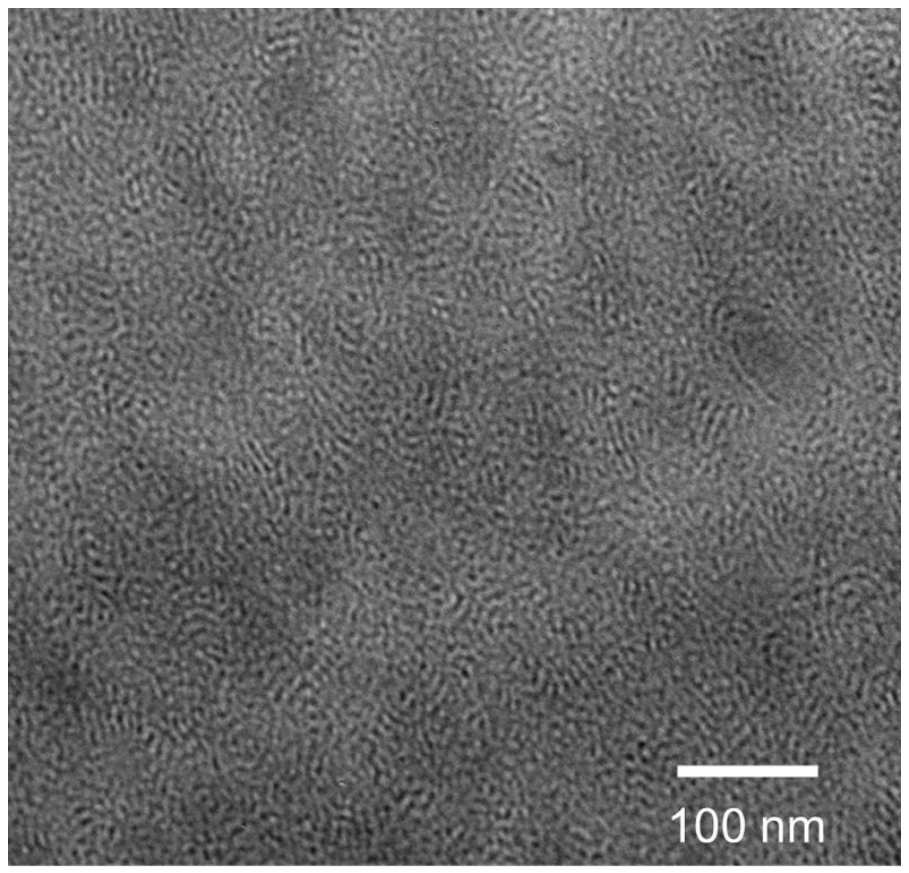

Figure S10. Large area TEM image of $\mathrm{PS}_{2.9}-\mathrm{PDMS}_{0.8}-146$. Disordered wormlike morphology is observed.

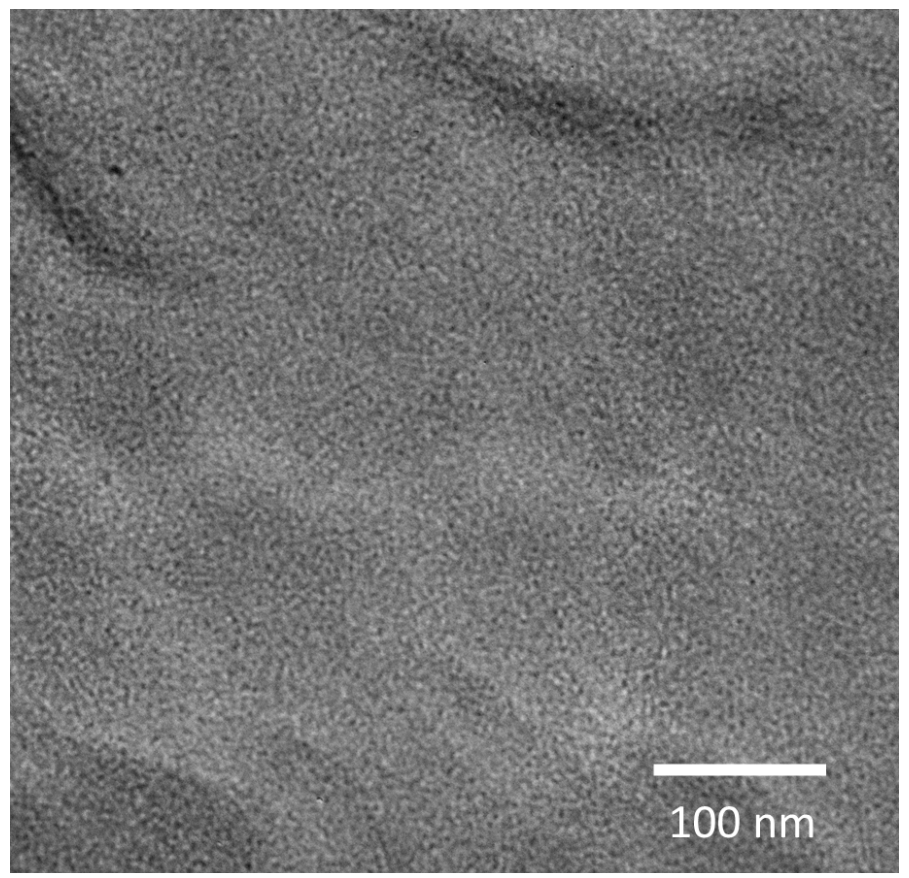

Figure S11. Large area TEM image of $\mathrm{PS}_{0.9}-\mathrm{PDMS}_{0.8^{-}}-58$. Sample is poorly microphase separated 


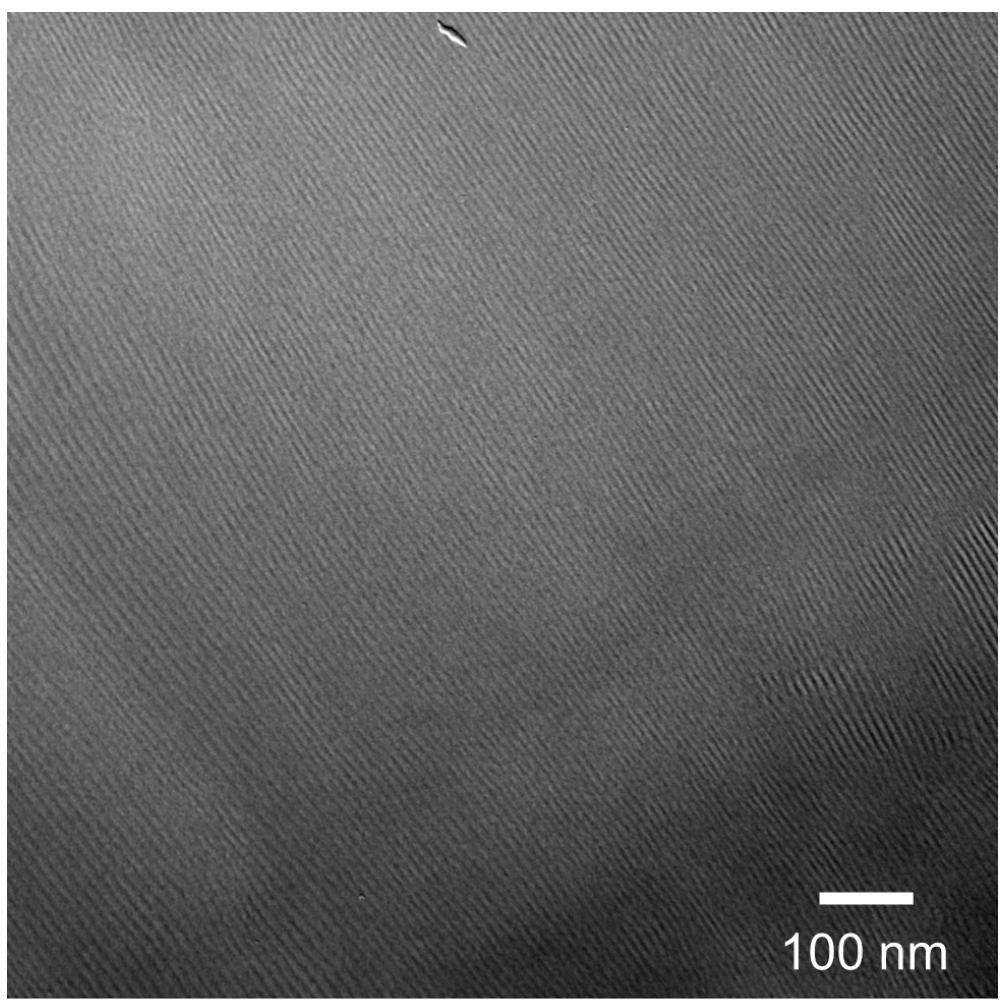

Figure S12. Large area TEM image of well-aligned $\mathrm{PS}_{4.7}-\mathrm{PDMS}_{4.8}-217$.

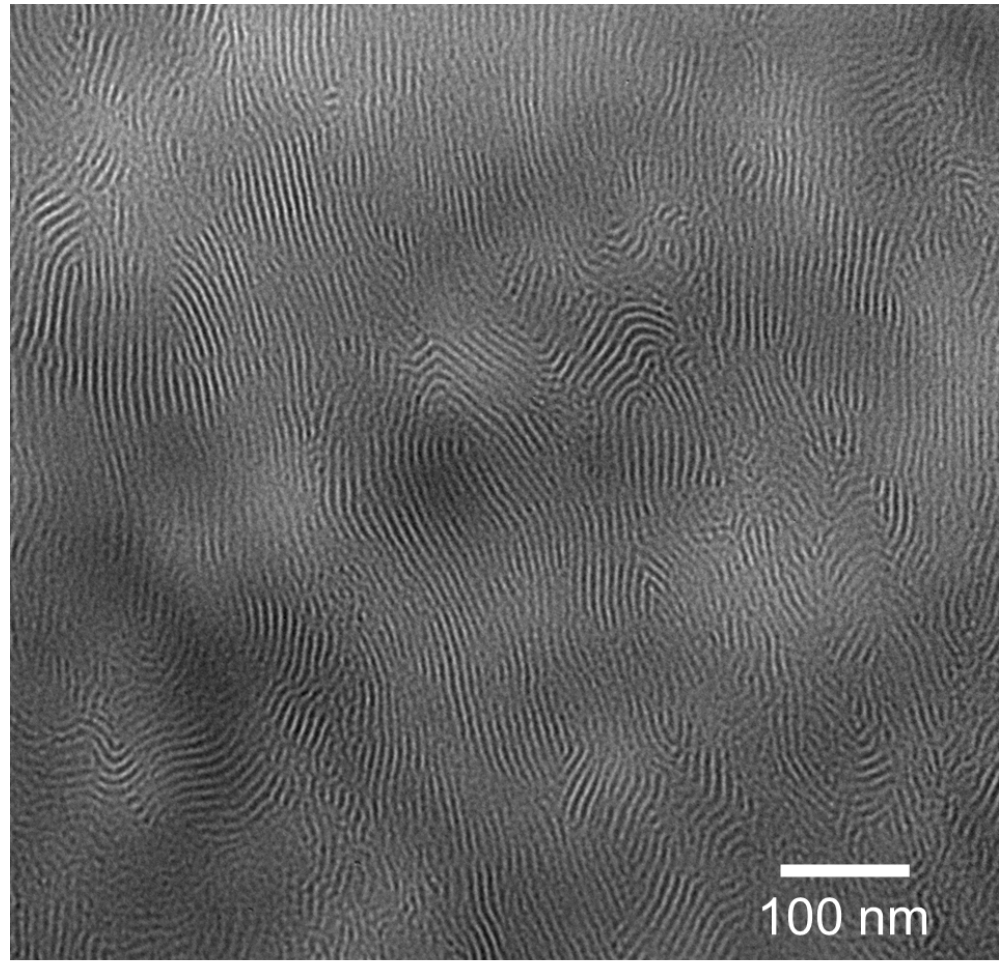

Figure S13. Large area TEM image of $\mathrm{PS}_{2.9}-\mathrm{PDMS}_{2.0}-306$. 


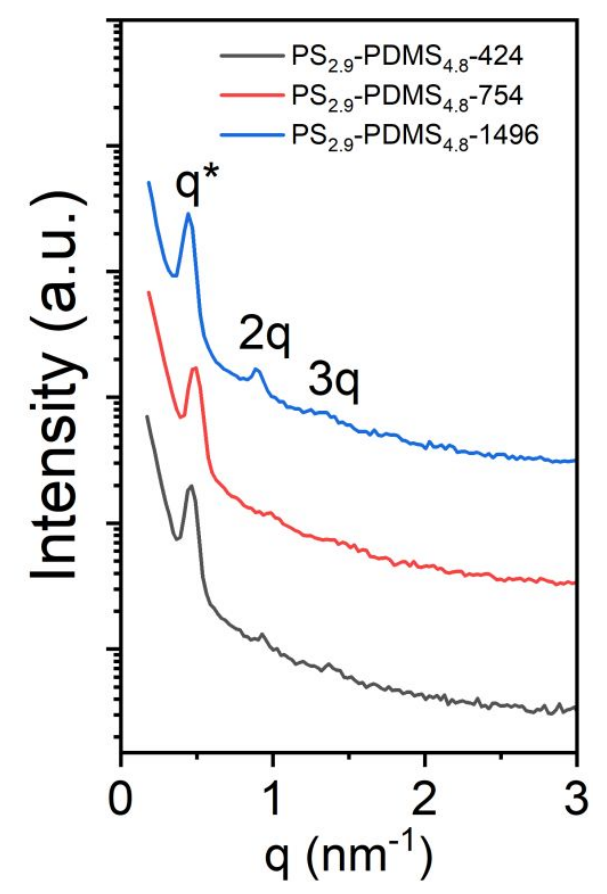

Figure S14. Additional SAXS profiles of BSCP samples with constant side chain lengths and increasing backbone length.

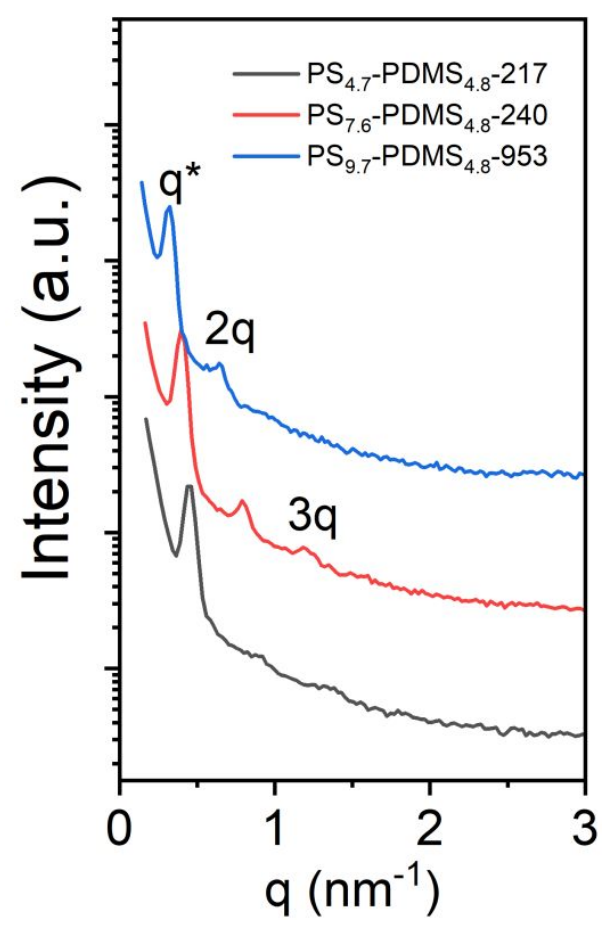

Figure S15. Additional SAXS profiles of BSCP samples with increasing PS side chain lengths 


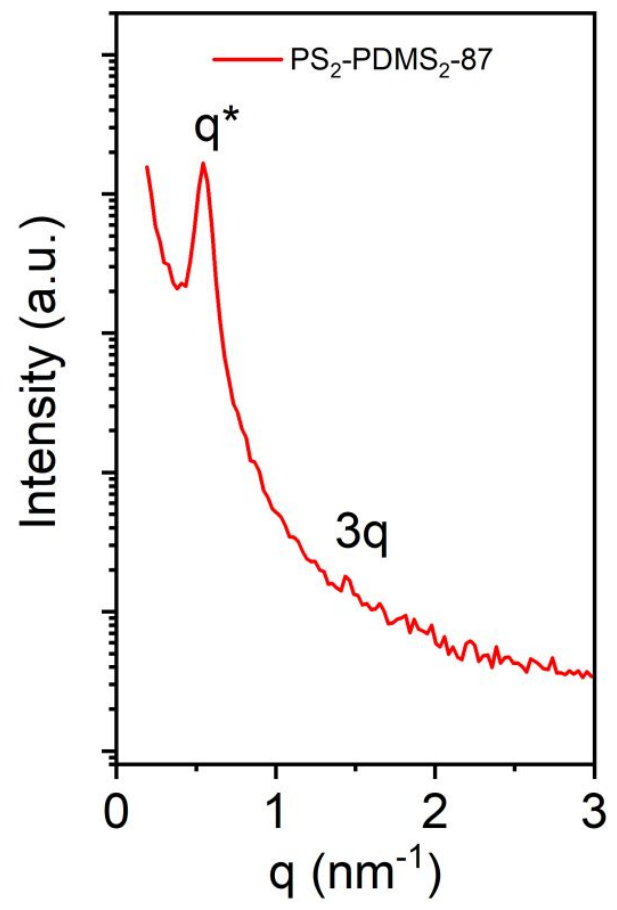

Figure S16. SAXS profiles for sample $\mathrm{PS}_{2}-\mathrm{PDMS}_{2}-87$.

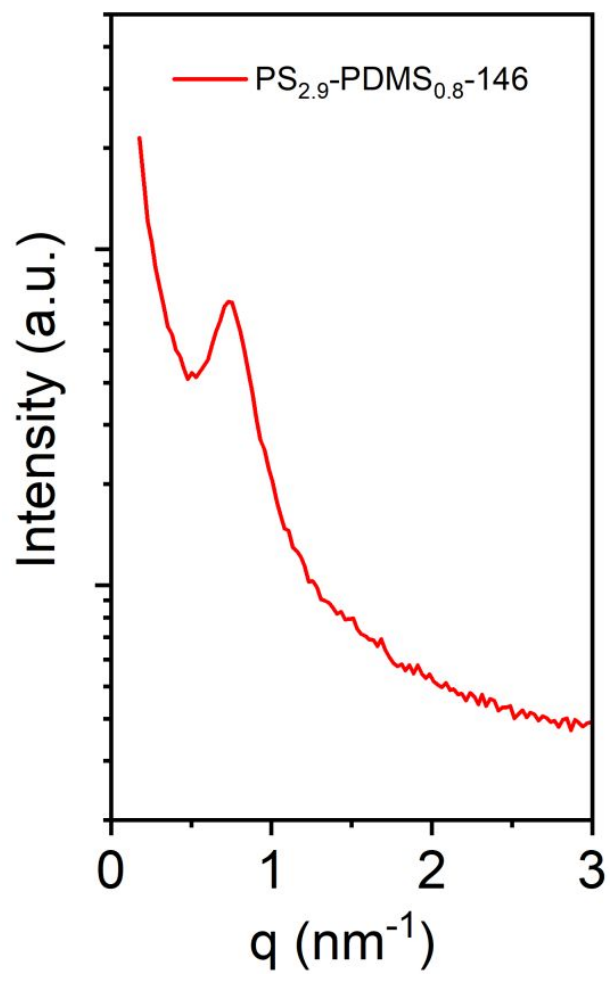

Figure S17. SAXS profiles for sample $\mathrm{PS}_{2.9} \mathrm{PDMS}_{0.8}-146$. 


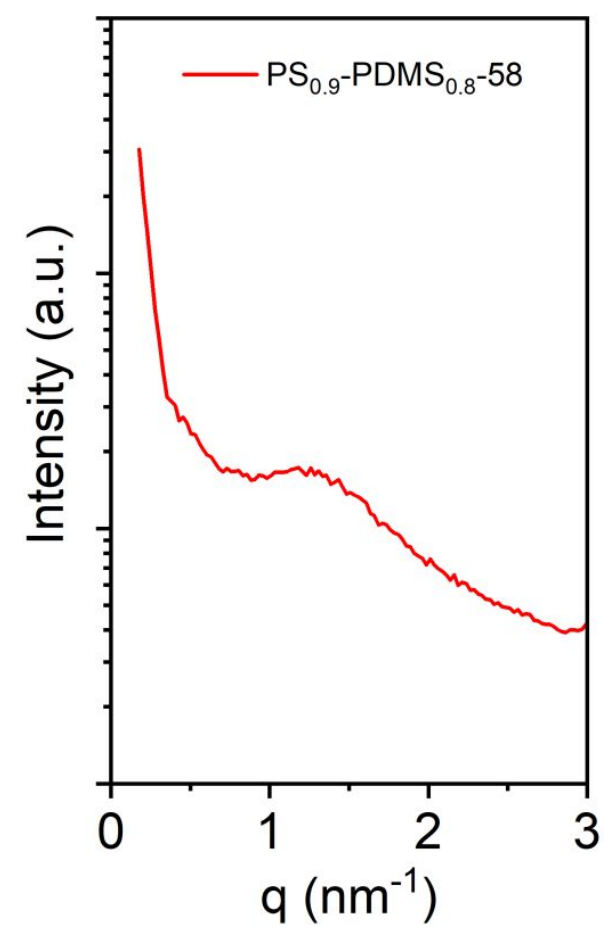

Figure S18. SAXS profiles for sample $\mathrm{PS}_{0.9}-\mathrm{PDMS}_{0.8}-58$.

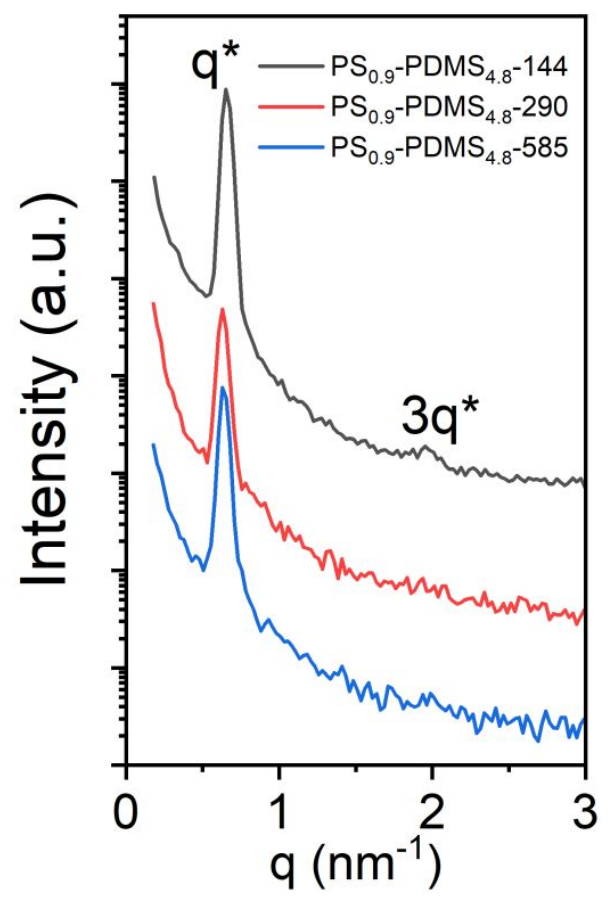

Figure S19. Additional SAXS profiles of BSCP samples with constant side chain lengths and increasing backbone length. 


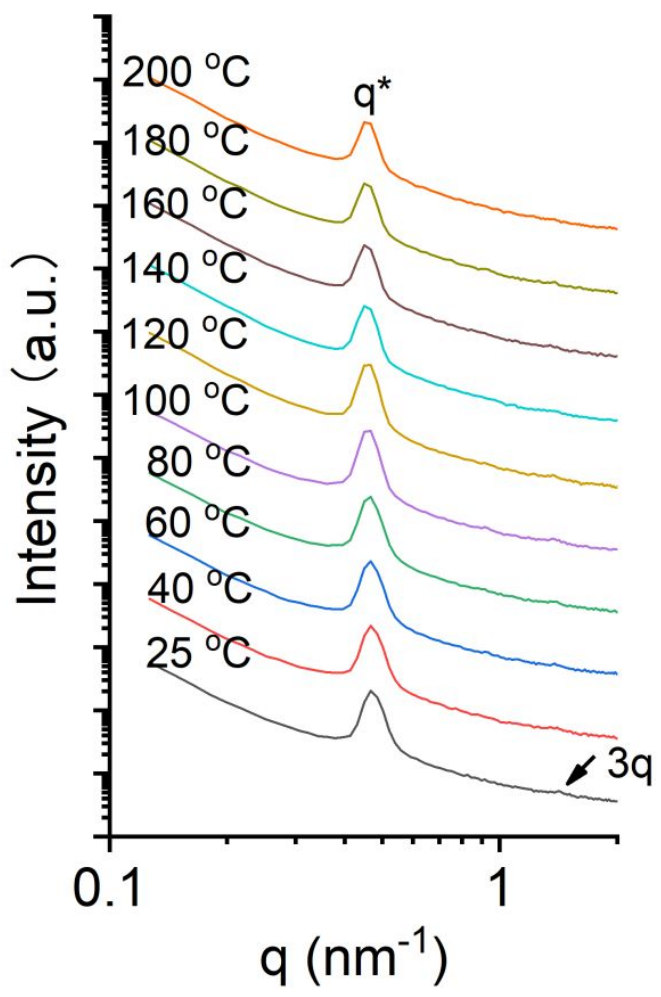

Figure S20. SAXS profile of $\mathrm{PS}_{2.9}-\mathrm{PDMS}_{4.8}-424$ with $f_{P S}=50 \%$ as a function of increasing temperature. Curves vertically shifted for clarity. Position and intensity of primary scattering peak $q^{*}$ is independent of temperature up to $T=200^{\circ} \mathrm{C}$.

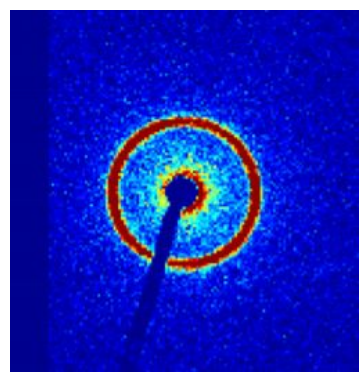

Figure S21. 2D SAXS patterns of the sample before thermal annealing. 
Table S1: Molecular characteristics of linear BCP analogs constructed from PS and PDMS side chains used for BSCP series

\begin{tabular}{ccccccc}
\hline $\begin{array}{c}\mathrm{PS} M_{w} \\
(\mathrm{~g} / \mathrm{mol})\end{array}$ & $\begin{array}{c}\mathrm{PDMS} M_{w} \\
(\mathrm{~g} / \mathrm{mol})\end{array}$ & $\mathrm{f}_{\mathrm{PS}}{ }^{\mathrm{a}}$ & $\mathrm{N}_{\mathrm{PDMS}}{ }^{\mathrm{b}}$ & $\mathrm{N}_{\mathrm{PS}}{ }^{\mathrm{b}}$ & $\mathrm{N}_{\text {Total }}$ & $\chi \mathrm{N}$ \\
\hline 2900 & 4800 & 0.36 & 28 & 65 & 93 & 27.8 \\
4700 & 4800 & 0.47 & 45 & 65 & 110 & 33.0 \\
7600 & 4800 & 0.59 & 73 & 65 & 138 & 41.4 \\
9700 & 4800 & 0.65 & 93 & 65 & 158 & 47.4 \\
900 & 4800 & 0.15 & 9 & 65 & 74 & 22.1 \\
2900 & 2000 & 0.57 & 28 & 27 & 55 & 16.5 \\
2900 & 800 & 0.77 & 28 & 11 & 39 & 11.6 \\
2000 & 2000 & 0.48 & 19 & 27 & 46 & 13.9 \\
900 & 800 & 0.51 & 9 & 11 & 19 & 5.8 \\
\hline
\end{tabular}

a Theoretical volume fraction of PS $\left(f_{\mathrm{PS}}\right)$ calculated using the molecular mass of PS and PDMS repeat units and density of $\rho_{P S}=1.05 \mathrm{~g} / \mathrm{cm}^{3}$ and $\rho_{P D M S}=0.96 \mathrm{~g} / \mathrm{cm}^{3}$. ${ }^{\mathrm{b}}$ Side chain length $\mathrm{N}_{P D M S}$ and $\mathrm{N}_{P S}$ calculated using monomeric molar mass and total molecular weight c Segregation strength calculated assuming $\chi=0.3$ at $25^{\circ} \mathrm{C}{ }^{3-4}$.

\section{References}

(1) Fei, H.-F; Yavitt, B. M.; Kopanati, G.; Watkins, J. J.: Effect of side chain and backbone length on lamellar spacing in polystyrene-block-poly(dimethyl siloxane) brush block copolymers. J. Polym. Sci., Part B: Polym. Phys. 2019, 57, 691-699.

(2) Fei, H.-F.; Yavitt, B. M.; Hu, X.; Kopanati, G.; Ribbe, A.; Watkins, J. J.: Influence of Molecular Architecture and Chain Flexibility on the Phase Map of Polystyrene-blockpoly(dimethylsiloxane) Brush Block Copolymers. Macromolecules 2019, 52, 6449-6457.

(3) Ninago, M. D.; Satti, A. J. ; Ciolino, A. E. ; Vallés, E. M. ; Villar, M. A. ; Vega, D. A. ; Sanz, A.; Nogales, A.; Rueda, D. R.: Synthesis and Morphology of Model PS-b-PDMS Copolymers. Journal of Poymer Science Part A: Polymer Chemistry 2010, 48, 3119-3127.

(4) Chu, J. H.; Rangarajan. P.; Adams, J. L.; Register, R. A. : Morphologies of Strongly Segregated Diblock Copolymers. . Science 1995, 36, 1569-1575. 